\title{
Microwave Dielectric Property Retrieval from Open-Ended Coaxial Probe Response with Deep Learning
}

This paper was downloaded from TechRxiv (https://www.techrxiv.org).

\section{LICENSE}

CC BY-NC-SA 4.0

SUBMISSION DATE / POSTED DATE

$12-11-2021 / 12-11-2021$

\section{CITATION}

Aydinalp, Cemanur; Joof, Sulayman; Akinci, Mehmet Nuri; Akduman, Ibrahim; Yilmaz, Tuba (2021): Microwave Dielectric Property Retrieval from Open-Ended Coaxial Probe Response with Deep Learning. TechRxiv. Preprint. https://doi.org/10.36227/techrxiv.16992394.v1

$\mathrm{DOI}$

10.36227/techrxiv.16992394.v1 


\title{
Microwave Dielectric Property Retrieval from Open- Ended Coaxial Probe Response with Deep Learning
}

\author{
Cemanur Aydinalp, Sulayman Joof, Mehmet Nuri Akinci, \\ Ibrahim Akduman, Tuba Yilmaz
}

\begin{abstract}
This work presents a technique for dielectric property retrieval through Debye parameter reconstruction from open-ended coaxial probe (OECP) response. Debye parameters were obtained with the application of a deep learning (DL) model to the reflection coefficient response of the OECP when terminated with a material under test. The OECP was modelled with the well-known admittance technique from 0.5 to $6 \mathrm{GHz}$ with $20 \mathrm{MHz}$ resolution. A dataset was generated using the admittance technique and obtained data was utilized to design the DL model. As part of the standard procedure, the dataset was separated to train, validate, and test parts by allocating the $80 \%, 10 \%$, and $10 \%$ of the dataset to each section, respectively. Obtained percent relative error for Debye parameters were $1.86 \pm 3.01 \%, 3.33 \pm 9.52 \%$, and $2.07 \pm 7.42 \%$ for $\epsilon_{s}, \epsilon_{\infty}$ and $\tau$, respectively. To further test the constructed DL model, OECP responses were measured at the same frequency band when it was terminated with five different standard liquids, four mixtures, and a gel-like material. Reconstructed Debye parameters from the DL model were used to retrieve the complex dielectric properties and obtained results were compared with the literature data. Obtained mean percent relative error was ranging from $1.21 \pm 0.06$ to $10.89 \pm 0.08$ within the frequency band of interest.
\end{abstract}

Index Terms-Complex permittivity, Debye parameters, deep learning, open-ended coaxial probe, admittance model.

\section{INTRODUCTION}

$\mathbf{M}$ ICROWAVE dielectric properties (DP), also referred to as complex permittivity, are critical system design parameters for the development of microwave diagnostic, imaging, treatment devices, and RF/microwave circuits. Therefore, inaccurate determination of the complex permittivity can render information resulting in poor system reliability. Based on the nature of the sample and frequency of interest different techniques can be employed to measure the DP. One such widely utilized technique is open-ended coaxial probe (OECP) method. The technique is used for the complex permittivity characterization of many different materials including but not limited to liquids, biological tissues, ice, and concrete [1], [2], [3], [4], [5], [6]. Provided that the full contact between the material under test (MUT) and the probe aperture is ensured, the method does not require strict sample preparation procedures. Additionally, the OECP technique is capable of performing wideband measurements of the MUTs complex

This work was supported by the Scientific and Technological Research Council of Turkey under grant agreement 118S074 and the European Union's Horizon 2020 research and innovation program under the Marie SklodowskaCurie grant agreement No 750346 .

The authors are with the Laboratory for Medical Device Research, Development and Application, Istanbul Technical University, Istanbul, 34469, TURKEY (e-mail: cemanuraydinalp@gmail.com) permittivity. While these advantages make the method a widely preferred tool for complex permittivity characterization, the OECP method is known to suffer from large measurement error. Commercially available probes report $\pm 5 \%$ accuracy under ideal conditions [7]. It is reported that the measurement error can increase up to $30 \%$ [8]. Sources of error include but not limited to the mathematical approach, calibration degradation, sample heterogeneity. Since the DP are not measurable quantities, complex permittivities are derived from the other measurable quantities. Thus, the mathematical approach can be a major contributor to the measurement error. Addressing the mathematical approach can aid in the improvement of the measurement accuracy of the OECP technique.

In the OECP technique, reflected signals from the MUT are measured and converted to the complex permittivity. Different mathematical approaches have been implemented to retrieve the complex permittivity from measured reflection coefficients including the full-wave method and the equivalent circuit model [9], [10]. Full-wave analysis provides higher accuracy results for wideband but suffers from poor convergence and slow computing performances when processing large quantities of data. The equivalent circuit model provides faster computing; however, suffers from low accuracy and limited bandwidth [11], [12], [13].

In [14], an admittance model is proposed to mathematically represent the relationship between the MUT complex permittivity and the measured reflection coefficients at the probe aperture. Different solution approaches including the quasi-static approximation, Taylor Series approach, stochastic approach, and particle swarm optimization (PSO) were proposed in the literature to retrieve the complex permittivity from the admittance model [11], [15], [5], [16], [17], [18]. While iterative solution techniques can converge under certain constraints, such as being valid until a certain probe diameter, heuristic approaches are likely to be stuck in a local minima. Moreover, these methods have high computational complexity; for non-linear inverse estimation problems, they reach the solution either (i) in an iterative manner by means of linear or quadratic approximations of the problem at each step or (ii) solving the forward problem many times for each estimation. In contrast to these approaches, the deep neural network model proposed in this work offers a non-iterative and nonlinear solution of the inverse estimation problem by directly learning the non-linearity of the problem from the data generated with the admittance model [19]. Moreover, after the training phase, it needs only simple operations (summations, multiplications and activation function evaluations) and it does not require a 
solution of forward problem, which makes it easily adoptable in hardware. To this end, deep neural networks have been successfully employed in the literature for high dimensional microwave modelling, such as extracting the parameters of microwave filters [20]. Therefore, we believe that the deep neural networks can be utilized in similar nonlinear inverse problems in electromagnetic, to potentially improve the computation time and to reduce complexity of solution while enhancing the ease of implementation.

In this paper, we propose an alternative method to find Debye parameters which are used to represent the complex permittivity of materials for a wide frequency range. The Debye parameters are retrieved from the reflection coefficient of the probe through deep learning (DL) model designed by utilizing a synthetically generated dataset. The reflection coefficients were numerically calculated using the admittance model proposed in [14] and the designed DL model was then tested with the measurement data. Briefly, $276 \times 17732$ input dataset was generated corresponding to the $3 \times 17732$ output Debye parameters dataset. The scattering parameters of the probe were calculated between $0.5-6 \mathrm{GHz}$, with $20 \mathrm{MHz}$ steps. The dataset was divided into three sections, $80 \%$ was used for training and $10 \%$ was used for validation. After the training procedure had ended, the remaining $10 \%$ of the dataset was used for testing. Obtained percent relative errors of the proposed model were $1.86 \pm 3.01 \%, 3.33 \pm 9.52 \%$, $2.07 \pm 7.42 \%$ for prediction of the Debye parameters $\epsilon_{s}$ (static dielectric permittivity), $\epsilon_{\infty}$ (dielectric permittivity at high frequencies), and $\tau$ (relaxation time constant), respectively. Finally, the trained deep learning model was tested with measurement data and it was found that the retrieved complex dielectric parameters had a percent mean relative error of approximately less than $10 \%$.

In the literature, an artificial neural network model is proposed in [21] to compute the complex permittivity from the measured reflection coefficients for biomedical applications. In the proposed study [21], 102 experiment samples including standard liquids and biological tissues were measured in order to train, validate and test the neural network model. Prediction accuracy of $\pm 5 \%$ was obtained for the complex permittivity. The study in [21] was limited with collected data from the experiments; therefore, the predictions of the trained neural network model was restricted. Composing a large dataset through measurements that include different materials, frequencies, and probes is labor intensive and costly. To the best of authors' knowledge, this work presents the first large scale study performed by designing a deep learning model using numerically generated data. Also, this work proposes to retrieve the Debye parameters from the reflection coefficient response of the OECP. The contributions of the paper and the added advantages are as follows:

1) One main contribution of this work is generation of data-set for training the deep neural network, which estimates the Debye parameters. In particular, unlike to [21], the input and output dataset was not measured but synthetically generated by means of a calibration procedure. Designing such procedures is an important topic for inverse electromagnetic estimation problems
[22], since it produces large amount of data required to train the deep learning model. Therefore, a more robust model is built in this work since a wide range of Debye parameters and reflection coefficients can be conveniently generated.

2) Since the probe is theoretically modelled, the proposed method provides design flexibility and ability to generate dataset for any desired probe with different dimensions.

3) Generating synthetic data shortens the time required for the collection of training data. For example, $276 \times 17732$ input dataset mentioned above was generated approximately in 50 mins on a computer with 192 GB RAM, $2 \times$ Intel(R) Xeon(R) X5690 3.47 GHz CPU. Furthermore, due to the features of deep neural networks, very large datasets can be easily trained using high-performance computing platforms such as GPUs.

4) Synthetic training approach proposed here is also flexible in data type and data production method. For instance, the dielectric permittivity model can be changed (Cole-Cole, Debye or multipole Debye) simply by using the target model for complex permittivity calculation or the simulation method can be different (instead of using the admittance model [14], numerical electromagnetic software such as HFSS or CST can be employed).

5) Different from the verification of previously proposed dielectric retrieval methods, which is usually performed by measuring few standard liquids, this work presents a statistical verification of the DL-based dielectric property retrieval technique both with synthetically generated and measured data.

The remainder of this paper is organized as follows: Section II-A presents the admittance model. Process for synthetic dataset generation is explained in section II-B Measurement procedure and the experimental setup are given in section II-C The design of the deep learning model is presented in section II-D Results are given in section III and conclusions are drawn in section IV

\section{MAterial AND Methods}

In the following section, we first present the theoretical background for retrieving the Debye parameters from the reflection coefficient using the OECP and admittance model. Second, based on the theoretical model, the dataset was generated to train, validate, and test the deep learning model. Furthermore, to test the deep learning model with measurement data, experiments were conducted to acquire the reflection coefficients of standard liquids, mixtures, and a gellike material. Finally, the design process for the deep learning model to retrieve the Debye parameters from the reflection coefficient is given in detail.

\section{A. Admittance Model for The Open-Ended Coaxial Probe}

The configuration of the OECP consists of two concentric cylindrical conductors with a dielectric material placed between the conductors. When the OECP is utilized to obtain the dielectric properties of a MUT, the relation between the complex permittivity of MUT and the admittance of the probe 
is given by the classical admittance model as shown below in equation (1) [14]:

$$
\frac{Y(\epsilon)}{Y_{0}}=\frac{i k_{0}^{2} \epsilon_{c}}{k_{d} \ln (b / a)} \int_{0}^{\infty} \frac{\left[J_{0}(\zeta a)-J_{0}(\zeta b)\right]^{2}}{\zeta \sqrt{\zeta^{2}-k_{0}^{2} \epsilon_{c}}} d \zeta
$$

where $Y(\epsilon)$ represents admittance of the probe when terminated with MUT, $Y_{0}$ is the probe characteristic admittance, $a$ and $b$ represent the inner and outer radius of the conductors, respectively. $J_{0}(\cdot)$ is the first order Bessel function, $k_{d}$ denotes the wave number of the dielectric material (between conductors) and $k_{0}$ represents the free space wave number. $\epsilon_{c}$ is the complex dielectric permittivity of the MUT which can be expressed in terms of Debye parameters:

$$
\epsilon_{c}(\omega)=\epsilon_{\infty}+\frac{\epsilon_{s}-\epsilon_{\infty}}{1+i \omega \tau}
$$

where $\omega$ represents the angular frequency, $\epsilon_{s}$ is the static constant, $\epsilon_{\infty}$ is the infinite frequency dielectric constant and $\tau$ is the characteristic relaxation time. Debye parameter equation 2 can be used for generating the complex dielectric constant. The admittance of the probe can then be derived from the equation 1 .

As result of the impedance difference between the reference medium and the MUT, the reflection coefficient $\Gamma$ at the probe's aperture can be expressed in terms of the ratio between the admittance of the MUT $Y(\epsilon)$ and the probe characteristic admittance $Y_{0}$ :

$$
\frac{Y(\epsilon)}{Y_{0}}=\frac{1+\Gamma}{1-\Gamma}
$$

From the equation 3 corresponding reflection coefficient can be derived. However, it should be noted that this represents the calculated reflection coefficient and does not correspond to a measured reflection coefficient. For a measured reflection coefficient, there is a need to adjust the reference plane of measurement through calibration in order to utilize equation 3 for measurements. Therefore, a calibration procedure is required to acquire the reflection coefficient $\left(\Gamma_{c}\right)$ at the probe's aperture. Please note that $\Gamma_{c}$ will be used to denote the calibrated reflection coefficient throughout this paper. Suppose, $\rho$ represents the reflection coefficient obtained from the MUT and $\rho_{1}, \rho_{2}, \rho_{3}$ are the reflection coefficients corresponding to three known material terminations. The reflection coefficient at probe's aperture $\left(\Gamma_{c}\right)$ can be obtained from the measured reflection coefficients $\rho, \rho_{i},(i=1,2,3)$ with the following expression:

$$
\Gamma_{c}=\frac{\rho-S_{11}}{\rho S_{22}+S_{12} S_{21}-S_{11} S_{22}}
$$

where $S_{11}, S_{12}, S_{21}$ and $S_{22}$ represent the unknown scattering parameters of the network [8], which can be evaluated with the subsequent equations:

$S_{11}=\frac{\Gamma_{1} \Gamma_{2} \rho_{3}\left(\rho_{1}-\rho_{2}\right)+\Gamma_{1} \Gamma_{3} \rho_{2}\left(\rho_{3}-\rho_{1}\right)+\Gamma_{2} \Gamma_{3} \rho_{1}\left(\rho_{2}-\rho_{3}\right)}{\Gamma_{1} \Gamma_{2}\left(\rho_{1}-\rho_{2}\right)+\Gamma_{1} \Gamma_{3}\left(\rho_{3}-\rho_{1}\right)+\Gamma_{2} \Gamma_{3}\left(\rho_{2}-\rho_{3}\right)}$

$$
\begin{gathered}
S_{12} S_{21}=\frac{\left(\rho_{1}-S_{11}\right)\left(1-S_{22} \Gamma_{1}\right)}{\Gamma_{1}} \\
S_{22}=\frac{\Gamma_{1}\left(\rho_{2}-S_{11}\right)+\Gamma_{2}\left(S_{11}-\rho_{1}\right)}{\Gamma_{1} \Gamma_{2}\left(\rho_{2}-\rho_{1}\right)}
\end{gathered}
$$

where $\Gamma_{1}, \Gamma_{2}$, and $\Gamma_{3}$ are the calculated reflection coefficients of the known materials using equations (1) and (3). For a measurement from a MUT, once the scattering parameters are calculated from the given expressions above, equation 4 is used to calculate the reflection coefficient at the aperture.

\section{B. Procedure for Obtaining the Deep Learning Dataset}

In this section, the expected reflection coefficient $(\Gamma)$ due to material's complex permittivities $\left(\varepsilon_{c}\right)$ is calculated using the admittance model. To do so, Debye parameters corresponding to a wide range of complex permittivity values were systematically generated. Note that the Debye parameters are frequently used for mathematical modelling of frequency dispersive complex permittivity. The theoretical relation between the Debye parameters and reflection coefficient at the probe's aperture can be expressed using equations (1), 2, and (3). Using any given set of Debye parameters $\left(\epsilon_{s}, \epsilon_{\infty}, \tau\right)$, the corresponding reflection coefficient $(\Gamma)$ at the probe's aperture can be directly calculated. The following steps were implemented to generate the required dataset. First, Debye parameters were generated by varying $\epsilon_{s}$ from 5 - 120 units, $\epsilon_{\infty}$ from 1 - 10 units, $\tau$ from $20-180$ ps with constant step size of $\frac{5}{\sqrt[3]{2}}, \frac{1}{\sqrt[3]{2}}$ and $\frac{4}{\sqrt[3]{2}}$, respectively. The step sizes were determined by trial and error. In this trial and error process the point that we noticed is to divide the output space (the space spanned by $\epsilon_{s}-\epsilon_{\infty}-\tau$ ) into equal pieces as much as possible in all three parameters. Next, generated Debye parameters were combined and a total of 17748 Debye parameter sets were obtained. Then, the complex dielectric properties were produced using Debye parameter sets. The frequency range was selected from 0.5 to $6 \mathrm{GHz}$ with $20 \mathrm{MHz}$ resolution (a total of 276 frequency points). Synthetically generated complex permittivity values were then placed in equation (1) to obtain the corresponding admittance. It should be noted that the dimensions of the probe ( $a$ and $b$ in equation (1) were chosen based on the commercially available Dielectric Assessment Kit (DAK). The outer radius of the probe (a) is $1.75 \mathrm{~mm}$ and inner radius (b) is $0.465 \mathrm{~mm}$ [23]. Finally, equation (3) was used to obtain the reflection coefficient. Through the application of this process, a dataset composed of reflection coefficients and corresponding Debye parameters was obtained. The dimensions of the dataset were $276 \times 17748(\Gamma)$ matrix (input of the deep learning model) corresponding to $3 \times 17748$ Debye parameters (output of the deep learning model).

\section{Experimental Setup and Protocol for Reflection Coefficient Measurements}

Since the deep learning algorithm is modelled based on the generated theoretical data, it is critical to evaluate the model's performance with real measurements. Therefore, an 


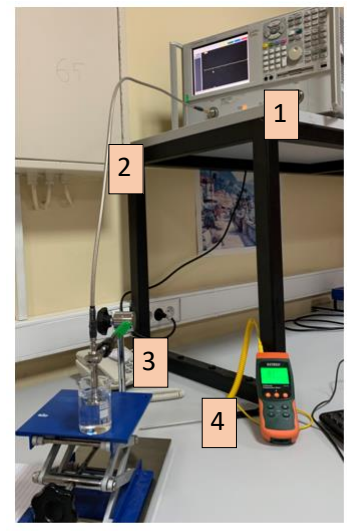

(a)

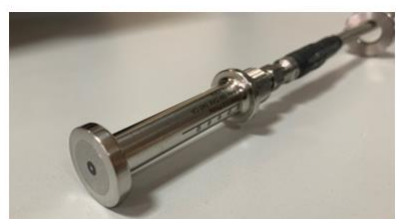

(b)

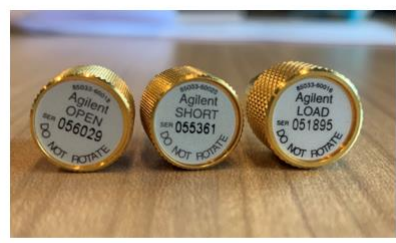

(c)
Fig. 1. Experimental setup and its components. (a) Experiment setup 1. N5230A PNA Series Network Analyzer, 2. RF cable, 3. sample: standard liquid and 4. EXTECH thermometer, (b) DAK 3.5 probe, and (c) mechanical calibration kit.

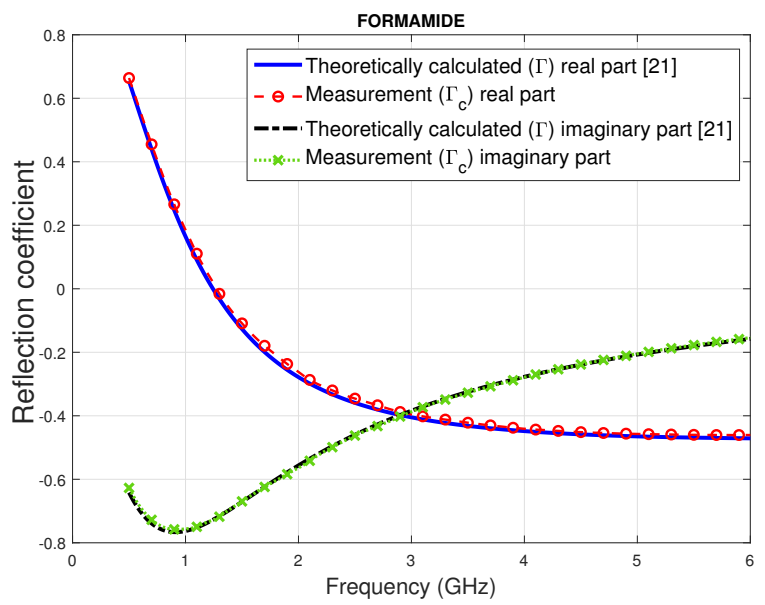

Fig. 2. Comparison of theoretically calculated reflection coefficient of formamide based on Debye parameters [24] and calibrated reflection coefficient obtained from measurements.

experiment setup shown in Fig. 1 (a) was used to collect the reflection coefficient responses of the probe with standard pure liquids. The experiment setup consisted of N5230A PNA Series Network Analyzer (Santa Clara, CA, USA) and the commercial Speag DAK $3.5 \mathrm{~mm}$-diameter open-ended coaxial probe (Zurich, Switzerland), shown in Fig. 1 (a) and (b). The frequency range of the analyzer was set from 0.5 to $6 \mathrm{GHz}$ with $20 \mathrm{MHz}$ increments; that is, 276 frequency points consistent with the synthetically generated data. An IF bandwidth of $10 \mathrm{~Hz}$ was selected to reduce the error due to noise. The analyzer and probe were connected via an RF cable. Before connecting cable to the probe, the Agilent 85033E $3.5 \mathrm{~mm}$ standard mechanical calibration kit (Santa Clara, CA, USA), as seen in Fig. 1 (c), was used for RF calibration with the aim to transfer the measurement reference plane to the end of the cable.
Obtaining the reflection coefficient for any MUT requires the calibration of the probe. The standard protocol for calibrating the OECP is a three-step process (open circuit, short circuit and distilled water). Probe calibration procedure for obtaining the reflection coefficient $\Gamma_{c}$ is given in section III-A To apply the given procedure in the section, measured reflection coefficients for the MUT $(\rho)$ and the reflection coefficients of three known materials $\left(\rho_{1}, \rho_{2}, \rho_{3}\right)$ were collected. The $\rho_{1}, \rho_{2}, \rho_{3}$ were measured by allowing the probe to radiate in free space (Open), terminating the probe's aperture with a copper strip (Short) and with a reference material (distilled water) respectively. The measured pure materials were DMSO, ethanol, ethylene glycol, formamide and methanol. The temperatures of the materials were $17.8 \pm 0.2^{\circ} \mathrm{C}$ at the time of the measurement. The mixtures were prepared via volume fractions: formamide (90\%)-1-buthanol (10\%), formamide (70\%)1-buthanol (30\%), DMSO (23\%)-ethanol (77\%) and DMSO (54\%)-1-butanol (\%46) at temperatures of $24.5 \pm 0.9^{\circ} \mathrm{C}$. Apart from the liquids/solutions, a gel-like material was prepared by mixing deionized water $(15 \mathrm{ml})$ and triton X-100 (15 $\mathrm{ml})$ at $23.5^{\circ} \mathrm{C}$. Each measurement, (3 reflection coefficients for calibration step and the measurement of the samples) were repeated five times for statistical verification purposes. As an example of the consistency between the theoretical calculation and calibration, Fig. 2 depicts the comparison of the theoretically calculated $\Gamma$ and the $\Gamma_{c}$ obtained from the calibrated reflection coefficient of formamide. From this figure, it can be concluded that the measured and theoretically generated data from the Debye parameters of formamide are in good agreement.

\section{Deep Learning Model Design}

Recently, many different studies have adopted deep learning to model nonlinear input-output relationships. This work presents the design of a deep neural network with 5 hidden layers as seen in Fig. 3 The inputs of the network were the real and imaginary parts of the reflection coefficients. As described in section II-B the input dataset was generated through the admittance model [14]. Before training the network, as a constraint, generated data with $\epsilon_{s}<\epsilon_{\infty}$ were omitted from the data set since it is known that most materials possess $\epsilon_{s}>\epsilon_{\infty}$ property. This approach shrank the dataset to $276 \times 17732$ reflection coefficients as input data and $3 \times 17732$ Debye parameters as the corresponding output data. Next, the dataset was divided into three sections, $80 \%$ was used for training and $10 \%$ was used for validation. After the training procedure was ended, the remaining $10 \%$ of the synthetically generated dataset was used for testing the designed network. Further, validation of the designed network was performed with the measured reflection coefficient data collected from a number of well-known liquids. As explained in section III-C, in order to collect a measurement dataset compatible with the synthetically generated data, reflection coefficients of the commercially available DAK probe were measured between 0.5 to $6 \mathrm{GHz}$, with steps of $20 \mathrm{MHz}$. Thus, the obtained number of input parameters and the frequencies were consistent with the synthetic dataset. 
The strategy of setting the neural network can be summarized as follows:

1) Normalize the output parameters with respect to its maximum value. For this study, $\epsilon_{s}, \epsilon_{\infty}$ and $\tau$ were normalized by 120,10 , and $180 \mathrm{ps}$, respectively. This approach was applied in order to increase the numerical stability of training.

2) Number of inputs is determined by the number of frequencies for which the measurements are performed. (For this study, the number of inputs was $2 \times 276$ since the input parameters are complex numbers.) The number of outputs is the number of parameters that is to be extracted. Note that in this work, the dielectric properties (DP) is mathematically represented with the Debye equation and the number of Debye parameters is three; thus, the number of outputs were three. The design can be started with a single hidden layer, whose size is determined as $B^{p_{i}}, B, p_{i} \in N$ (i.e. integer powers of an integer) where $i$ is the order of the related layer. In this study, $B=2$ and we started with $p_{1}=7$.

3) Assume that there are $L$ many hidden layers, let the training error for this case is $E_{t, L}$ and validation error for this case is $E_{v, L}$. If $E_{t, L} \approx E_{v, L}$ then increase the number of free parameters of neural network either by choosing $p_{L}:=p_{L}+1$ or by setting $p_{L+1}>0, p_{L+1} \in N$.

4) If $E_{t, L+1} \approx E_{v, L+1}<E_{t, L} \approx E_{v, L}$, then go step 3 . If $E_{t, L+1}<E_{t, L}$ but $E_{v, L+1} \approx E_{v, L}$ then the neural networks start to overfit the data; thus, go to the previous state and choose the final design with $L$ layers.

Following the guidelines given above, the deep neural network model shown in Fig. 3 was designed. Please note that using batch normalization (BN) and Relu layer (ReLu) after each dense layer (DN), improves the model. Thus, after each dense layer, these two blocks were applied to the data subsequently. Finally, a regression layer (RL) was applied. The given model was trained on GPU using Matlab's Deep Neural Network Toolbox on a computer with 32 GB RAM, 16 GB Intel(R) UHD Graphics $630 \mathrm{GPU}, 2.6 \mathrm{GHz}$ CPU. In the training, maximum of 200 epochs were applied to the model and Adam optimizer was used with least mean square error cost function. The training lasted approximately 10 mins and at the end of training the model had a loss of 0.02 for both training and validation data, indicates that the model did not overfit. Finally, to further show that the model can represent the nonlinear relationship between reflection coefficients and Debye parameters, test data (10\% of the synthetic dataset) was processed by the trained network. For each case, the predicted Debye parameters were compared with the actual values and the percent relative error for each parameter was computed $\left(\frac{\mid \text { Predicted }-E x a c t \mid}{\mid \text { Exact } \mid} \times 100 \%\right)$. There were approximately 1700 sample in the test data and the histograms of percent relative error for each parameter are given in Fig. 4 Additionally, the mean and standard deviation of the percent relative errors of the test data are given in Table I. As can be seen from these results, the designed model can accurately determine the Debye parameters.

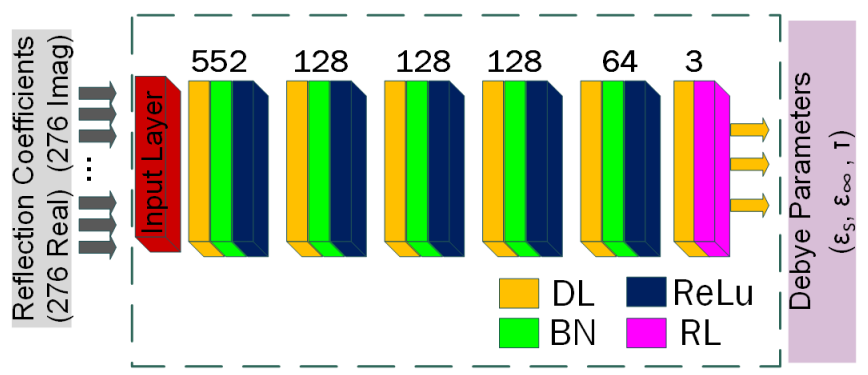

Fig. 3. Designed deep learning model with dense layer (DN), batch normalization (BN), Relu layer (ReLu) and regression layer (RL) for predicting Debye parameters from reflection coefficients.
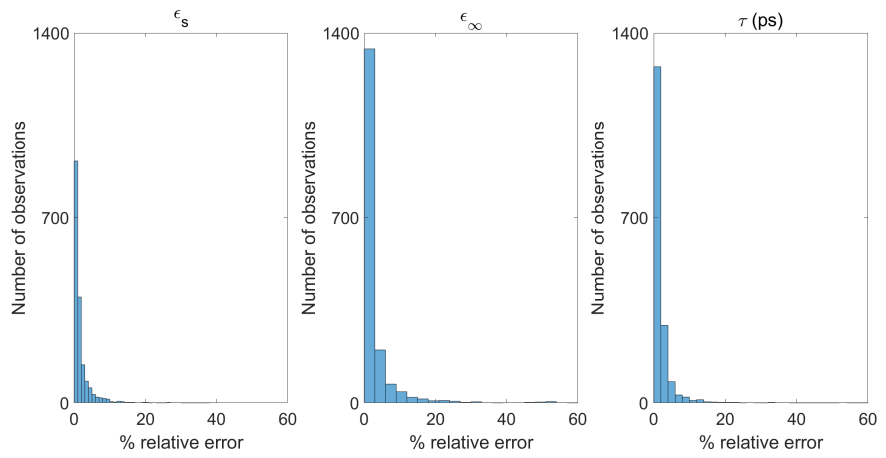

Fig. 4. Histograms of percent relative errors for each output parameter $\left(\epsilon_{s}\right.$, $\epsilon_{\infty}$ and $\tau$ ) obtained for 1700 samples in the test set (10\% of the synthetic dataset).

\section{RESUlTS AND Discussion}

To further test the optimized deep learning model with measured data, a dataset was generated by using 5 standard liquids; namely, dimethyl sulfoxide (DMSO), ethanol, ethylene glycol, formamide, and methanol; and 4 mixtures. To compose the dataset, 5 reflection coefficient measurements with the commercially available DAK probe were collected from each of the liquids/mixtures, and the calibration standards; that is, open, short and distilled water. Then, 5 measurements for each material were combined with the calibration (open+short+water+material) to obtain a total of 625 different measurement. Thus, the obtained test datasets generated from experiments were composed of $5 \times 625$ measurements for standard liquids and $4 \times 625$ measurements for mixtures. Apart from these two dataset, the deep learning model was tested with a gel-like material with the same data collection process.

The first dataset $(5 \times 625)$ was fed to the optimized deep neural network and the mean of the obtained network outputs;

TABLE I

THE MEAN AND STANDARD DEVIATION OF THE PERCENT RELATIVE ERRORS FOR DEB YE PARAMETERS $\left(\epsilon_{s}, \epsilon_{\infty}\right.$ AND $\left.\tau\right)$ OF THE TEST SET $(10 \%$ OF THE SYNTHETIC DATASET)

\begin{tabular}{|c|c|c|}
\hline Debye parameters & Mean (\%) & Std dev. (\%) \\
\hline$\epsilon_{s}$ (units) & 1.86 & 3.01 \\
\hline$\epsilon_{\infty}$ (units) & 3.33 & 9.52 \\
\hline$\tau$ (ps) & 2.07 & 7.42 \\
\hline
\end{tabular}


TABLE II

COMPARISON OF DEBYE PARAMETERS GIVEN IN LITERATURE AND MEAN OF THE RETRIEVED DEB YE PARAMETERS FROM THE DESIGNED NEURAL NETWORK (NN) FOR STANDARD LIQUIDS. MPE \pm SPE FOR THE CALCULATED COMPLEX PERMITTIVITY $\left(\varepsilon_{c}\right)$ FROM RETRIEVED DEBYE PARAMETERS WITH RESPECT TO LITERATURE VALUES FROM 0.5 TO $6 \mathrm{GHZ}$

\begin{tabular}{|l|c|c|c|c|c|c|c|}
\hline \multirow{2}{*}{ Standard Liquids } & \multicolumn{2}{|c|}{$\epsilon_{s}$ (units) } & \multicolumn{2}{c|}{$\epsilon_{\infty}$ (units) } & \multicolumn{2}{c|}{$\tau$ (ps) } & $\epsilon_{c}$ \\
\cline { 2 - 8 } & Ref. & NN-results & Ref. & NN-results & Ref. & NN-results & MPE \pm SPE (\%) \\
\hline Dimethyl sulfoxide [25] & 47.13 & 46.81 & 6.8 & 4.98 & 21.6 & 19.86 & $1.21 \pm 0.06$ \\
\hline Ethanol [26] & 25.07 & 22.82 & 4.2 & 4.00 & 143.24 & 150.22 & $10.89 \pm 0.08$ \\
\hline Ethylene Glycol [27] & 35.85 & 37.34 & 4.23 & 4.79 & 99.62 & 115.14 & $6.08 \pm 0.02$ \\
\hline Formamide [24] & 111.8 & 109.23 & 6.9 & 5.26 & 42.00 & 42.9 & $3.68 \pm 0.03$ \\
\hline Methanol [26] & 33.64 & 37.01 & 5.7 & 4.94 & 53.05 & 57.21 & $8.53 \pm 0.02$ \\
\hline
\end{tabular}
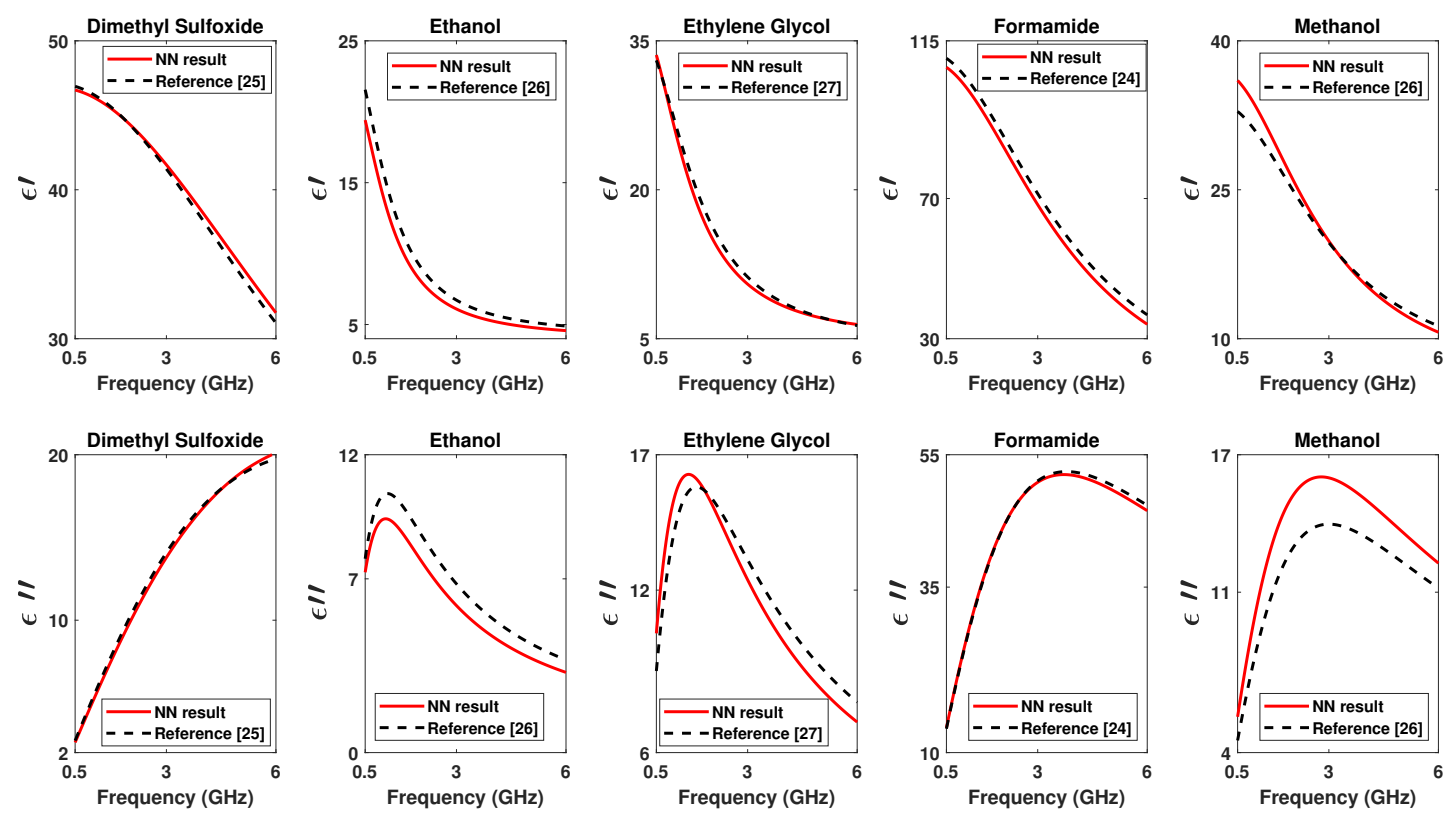

Fig. 5. Comparisons of calculated complex permittivities from the retrieved mean of Debye parameters and literature values of complex permittivities for five standard liquids: dymethyl sulfoxide, ethanol, ethylene glycol, formamide and methanol.
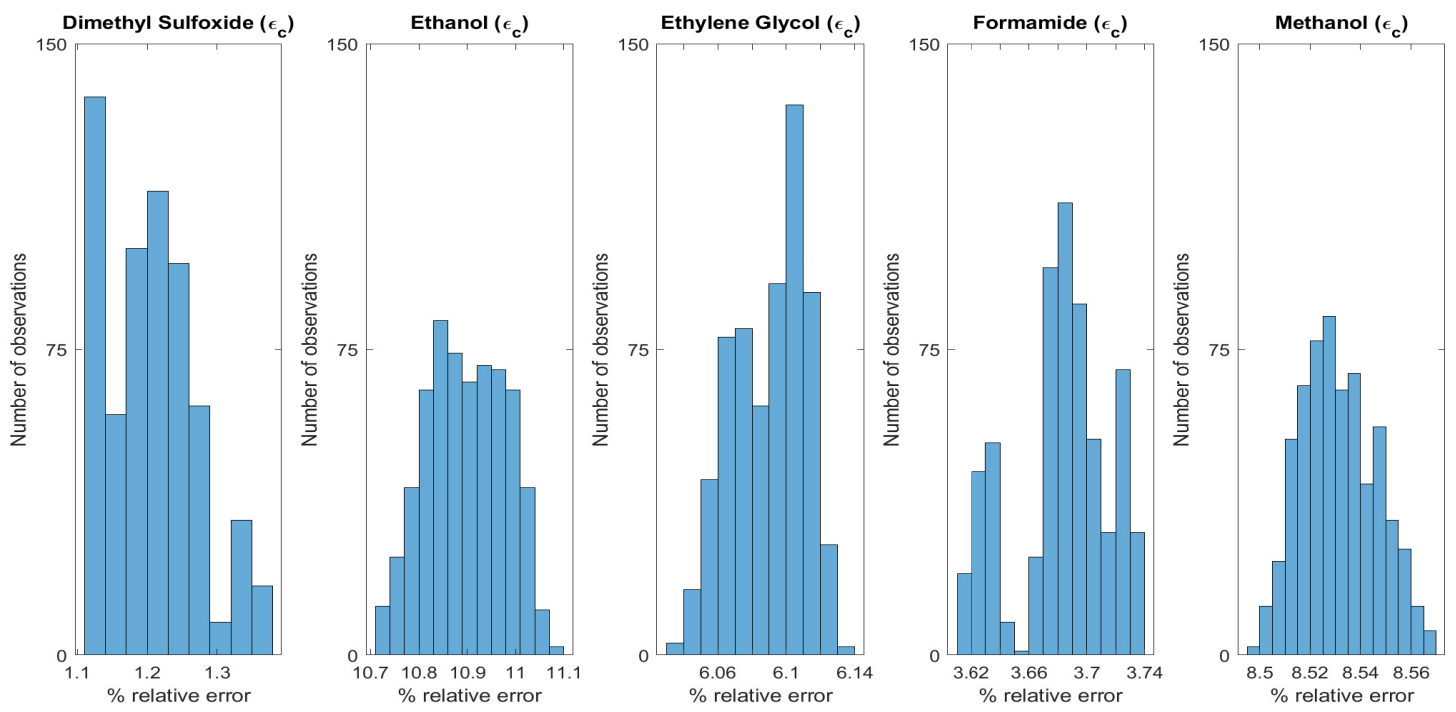

Fig. 6. The calculated $E_{\text {single }}$ of eq.87 of deep learning model by testing over 625 measurements for each standard liquid. 
TABLE III

COMPARISON OF DEBYE PARAMETERS GIVEN IN LITERATURE AND MEAN OF THE RETRIEVED DEBYE PARAMETERS FROM THE DESIGNED NEURAL NETWORK (NN) LIQUID MIXTURES. MPE \pm SPE FOR THE CALCULATED COMPLEX PERMITTIVITY $\left(\varepsilon_{c}\right)$ FROM RETRIEVED DEBYE PARAMETERS WITH RESPECT TO LITERATURE VALUES FROM 0.5 TO $6 \mathrm{GHZ}$

\begin{tabular}{|l|c|c|c|c|c|c|c|}
\hline \multirow{2}{*}{ Liquid Mixtures (Vol. frac. \%) } & \multicolumn{2}{|c|}{$\epsilon_{s}$ (units) } & \multicolumn{2}{c|}{$\epsilon_{\infty}$ (units) } & \multicolumn{2}{c|}{$\tau$ (ps) } & $\epsilon_{c}$ \\
\cline { 2 - 9 } & Ref. & NN-results & Ref. & NN-results & Ref. & NN-results & MPE \pm SPE (\%) \\
\hline Formamide (90) \& 1-Butanol (10) [28] & 101.0 & 100.69 & 5.0 & 2.64 & 41.1 & 43.9 & $5.9 \pm 0.18$ \\
\hline Formamide (70) \& 1-Butanol (30) [28] & 79.0 & 78.14 & 4.9 & 3.29 & 58.3 & 56.95 & $1.75 \pm 0.05$ \\
\hline * DMSO (23) \& Ethanol (77) [29] & 29.4 & 29.35 & 4.7 & 4.4 & 80.8 & 53.6 & $8.9 \pm 0.06$ \\
\hline ** DMSO (54) \& 1-Butanol (46) [29] & 34.1 & 33.83 & 4.3 & 4.43 & 51.5 & 39.11 & $4.83 \pm 0.02$ \\
\hline
\end{tabular}

* The parameter of $\beta$ equals 0.77 in Debye equation [29].

** The parameter of $\beta$ equals 0.84 in Debye equation [29].
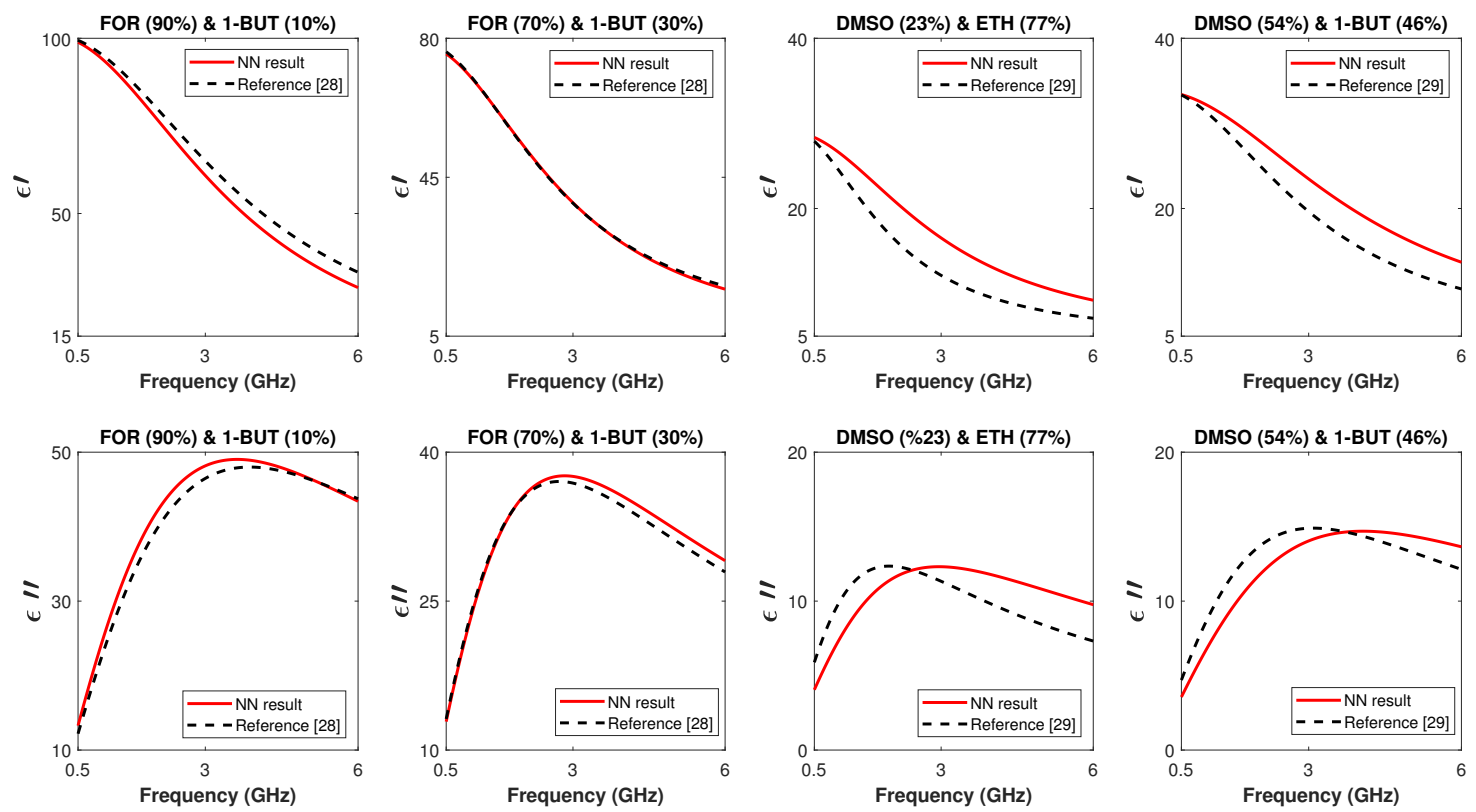

Fig. 7. Comparisons of calculated complex permittivities from the retrieved mean of Debye parameters and literature values of complex permittivities for three liquid mixtures: formamide (90\%) \& 1-butanol (10\%), formamide (70\%) \& 1-butanol (30\%), DMSO (23\%) \& ethanol (77\%) and DMSO (54\%) \& 1-butanol (46\%).
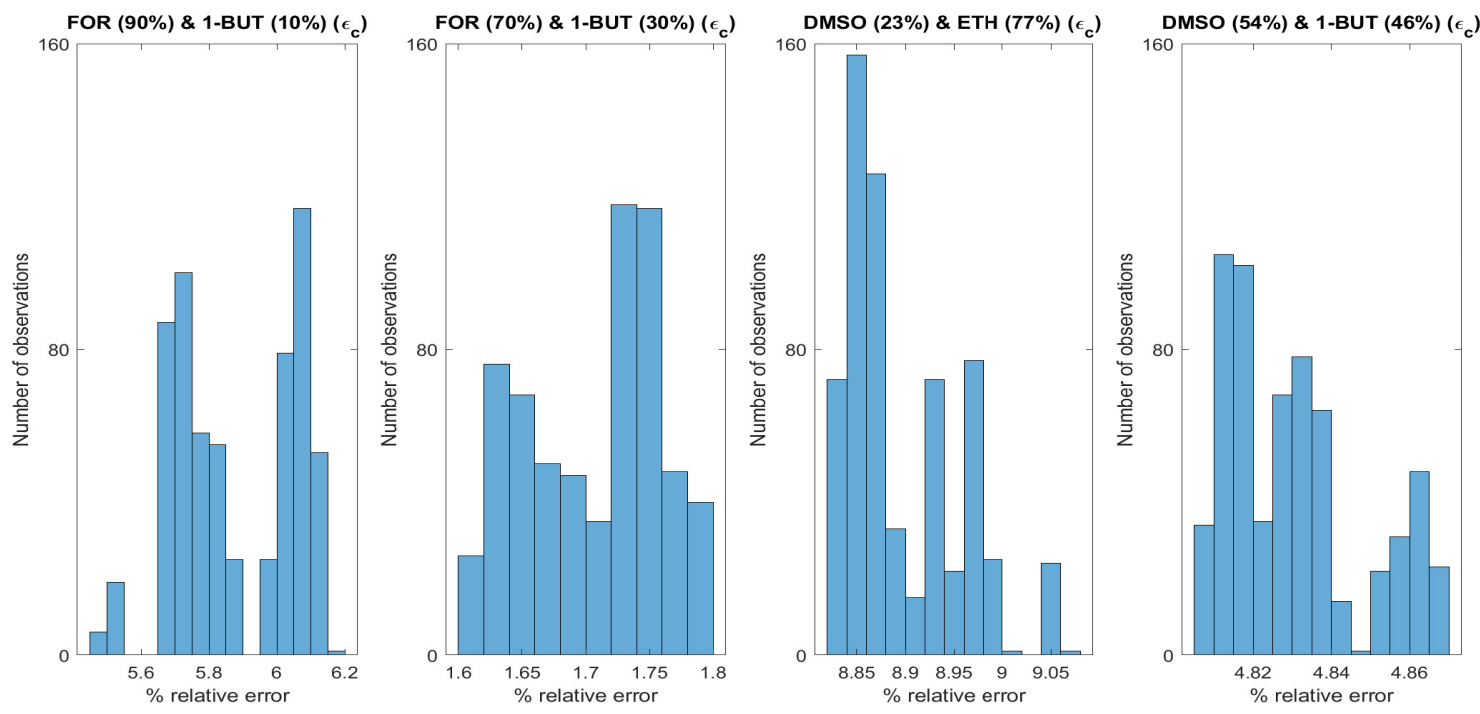

Fig. 8. The calculated $E_{\text {single }}$ of eq. 8 of deep learning model by testing over 625 measurements for each liquid mixture. 
that is, mean Debye parameters corresponding to the dataset and literature values for five standard liquids are listed in Table II Furthermore, the complex permittivity was computed from the retrieved Debye parameters using equation (2). The mean of percent relative error (MPE) and standard deviation of percent relative error (SPE) for the calculated complex permittivity from the retrieved Debye parameters are also given in Table II The MPE was calculated from $0.5-6 \mathrm{GHz}$ using the equation of:

$$
\begin{array}{r}
E_{\text {single }}=\frac{\sum_{\text {Freq }} \frac{\mid \text { Predicted }- \text { Exact } \mid}{\mid \text { Exact } \mid}}{276} \times 100 \\
M P E=\frac{\sum_{\text {Measurements }} E_{\text {single }}}{625}
\end{array}
$$

where 'Predicted' is the complex permittivity calculated from the Debye parameters acquired from the deep learning neural network model and 'Exact' is the complex permittivity computed using Debye parameters obtained from the literature. Similarly, the SPE is calculated as:

$$
S P E=\frac{\sum_{\text {Measurements }}\left(E_{\text {single }}-M P E\right)^{2}}{625}
$$

As seen in Table [I] all MPEs are below 11\%. Moreover, the SPEs for complex permittivity is below 0.08 units, which demonstrates that the deep learning model is robust enough to predict Debye parameters from reflection coefficients. In Fig. 5. a comparison of real $\left(\epsilon^{\prime}\right)$ and imaginary $\left(\epsilon^{\prime \prime}\right)$ parts of the complex permittivity calculated from the retrieved neural network Debye parameters and literature values are shown for five standard liquids from 0.5 to $6 \mathrm{GHz}$. To further investigate the accuracy of the deep learning model, the histograms for $E_{\text {single }}$ of eq. (8) is given in Fig. 6. The maximum $E_{\text {single }}$ was $11.1 \%$ for ethanol and the minimum $E_{\text {single }}$ was $1.1 \%$ DMSO.

The second test dataset $(4 \times 625)$ includes the prepared mixtures. The mean of the estimated Debye parameters from neural network (NN) corresponding to the dataset and the literature values are listed in Table III] Also, in the last column of Table III. the MPE and SPE for the calculated complex permittivity from the retrieved Debye parameters is given. The results from Table IIII indicate that the obtained MPEs for all the mixtures are below 8.9\%. Furthermore, the SPEs for complex permittivity is below 0.18 units. Comparison of the real $\left(\epsilon^{\prime}\right)$ and the imaginary $\left(\epsilon^{\prime \prime}\right)$ parts of the complex permittivity, which are computed from the retrieved Debye parameters and the literature, are shown in Fig. 7. The histograms $E_{\text {single }}$ in complex permittivity for each mixture are given in Fig. 8. The maximum $E_{\text {single }}$ for the calculated complex permittivity was $9.05 \%$ for the DMSO and ethanol mixture (volume ratio 2.3:7.7) and the minimum $E_{\text {single }}$ obtained from the formamide and 1-butanol mixture (volume ratio 7:3) is $1.6 \%$. As seen from the histograms, the error of prediction from the deep learning model has differed in a small range for each sample.

The obtained results are an indication that the proposed approach is robust and can be used to retrieve the dielectric properties of materials with the OECP technique. When this study is compared with the previously reported work in the literature, to the best of authors' knowledge, only one reported work carried out a study utilizing $\mathrm{NN}$ to retrieve complex permittivity with OECP response [21]. However, the reported study only used measurements; therefore, train and test data were restricted with the measurements and availability of the materials. Also, in [21], the complex permittivity values of methanol were calculated from 0.5 to $5 \mathrm{GHz}$ frequency range from three samples. The obtained real and imaginary values for methanol at $2.45 \mathrm{GHz}$ were approximately 24 and 15 units, respectively. The computed real and imaginary values from the Debye parameters of methanol retrieved from the deep learning algorithm proposed in this work at $2.46 \mathrm{GHz}$ are 23 and 15.9 units, respectively. To further expand the comparison, a gel-like solution containing $15 \mathrm{ml}$ Triton X-100 and $15 \mathrm{ml}$ de-ionized water was prepared. At $2.45 \mathrm{GHz},[21]$ reported 35 and 8 units for real and imaginary parts of DP, respectively. In this work, the retrieved real and imaginary DP values from the trained $\mathrm{NN}$ at $2.46 \mathrm{GHz}$ are 33.4 and 10.4 units, respectively. It should also be noted that, unlike [21], our work provides statistical analysis of 625 different combinations of methanol measurements.

In [25], the traditional approach for dielectric property characterization with iterative solution techniques was implemented. Furthermore, the Debye parameters of DMSO between $30 \mathrm{MHz}$ and $5 \mathrm{GHz}$ frequency band were reported with approximately $95 \%$ confidence level using linear interpolation via Monte Carlo modelling. The calculated MPE between obtained results in this work and [25] is $1.21 \pm 0.06$ for DMSO. As seen in Table [I] the maximum error was observed in the complex permittivity of ethanol. Instead of the single-pole Debye (3 parameters), the Debye- $\Gamma$ (4 parameters) can potentially offer a more suitable relaxation equation for ethanol as mentioned in [25]. In [27], the measurement of ethylene glycol was performed using the transmission line (TL) approach from 0.3 to $3 \mathrm{GHz}$. The Debye parameters were calculated from the complex permittivity by solving a system of nonlinear equations using MATLAB's fsolve function. The calculated Debye parameters for ethylene glycol differ from the work in [27] as much as 1.51 units, 0.56 units and $15.52 \mathrm{ps}$ for $\epsilon_{s}, \epsilon_{\infty}$ and $\tau$, respectively. These differences can be due to the different approaches used in obtaining the Debye parameters. In [24], the complex permittivity of formamide was measured from $10 \mathrm{MHz}$ to $70 \mathrm{GHz}$ frequency range and the best fit was represented by a single-Debye type dispersion. As seen in Table II the retrieved Debye parameters difference in [24] and this work is 2.57 units, 1.64 units and $0.9 \mathrm{ps}$ for $\epsilon_{s}, \epsilon_{\infty}$ and $\tau$, respectively. In [28], formamide and 1-butanol were mixed with different volume fractions. The measurements were taken over the frequency range of 0.2 to 13.5 GHz. In this work, two different mixtures were prepared in fractions of 9:1 and 7:3. The results in Table [III indicate that the $\mathrm{MPE} \pm \mathrm{SPE}$ is $5.9 \pm 0.18$ and $1.75 \pm 0.05$ for these mixtures. In another study [29], the dielectric properties of the mixture of DMSO and monohydric alcohol were analyzed. In this work, DMSO and ethanol mixture was prepared with a volume fraction of 2.3:7.7, corresponding to a molar fraction of $2: 8$. The results show that the MPE \pm SPE is $8.9 \pm 0.05$. Similarly, DMSO and 1-butanol mixture solution was prepared 
with a volume fraction of 5.4:4.6, that is molar fraction of $6: 4$. The obtained results indicate MPE \pm SPE of $4.83 \pm 0.02$. Consequently, the results obtained from this work match well with the literature data, which were obtained through different methods.

As a final example, the trained NN is compared with the brute force look up table (LUT) approach. The LUT has two problems: (i) it requires a data table to be held in memory and (ii) for each estimation, the points that are close to the data point has to be determined, which requires evaluating the distance between the data point and the points in the LUT. These two problems become worse with increasing the dimension of the measured data. In contrast to this, a trained NN consists of simple computations (multiplications, summations and activation function evaluations). From another perspective, LUT is similar to memorization, it tries to separate all instances of data points by dividing the output space into Voronoi cells with respect to a metric, while $\mathrm{NN}$ is akin to generalization (or vary much similar to learning with aid of a complicated formula). The goal of $\mathrm{NN}$ is to find a nonlinear function that can map the input to output. To further explore the difference, the DMSO example in Table III is re-estimated with the LUT approach. $80 \%$ of the dataset $(17732 \times 0.8$ S-parameters simulations, each of which includes 276 frequencies) are used to form a LUT. With the perspective defined above, we can think that there are $17732 \times 0.8$ many points in $R^{276 \times 2}$ in LUT. Then, the Euclidean distance between DMSO measurement in Table III and each data point in LUT is calculated and the Debye parameters of DMSO are assigned as the Debye parameters of the closest point in LUT. To assess the robustness of the LUT based method, it was evaluated 50 times (with different selections of $17732 \times 0.8$ many S-parameters simulations from 17732 simulations) and the average Debye parameters are estimated as $\epsilon_{s}=48.7$, $\epsilon_{\infty}=7.35$ and $\tau=24.0$ ps with an MPE of $3.37 \%$ (this was $1.21 \%$ for DMSO using $\mathrm{NN}$ as seen from Table III. In terms of computational speed, NN based method spends 3.8 ms on average, while LUT spends $24.0 \mathrm{~ms}$ on average for one single dielectric property estimation. From the point-view of memory consumption, the LUT needs much more resources than the NN, since it needs a large table $(276 \times 17732 \times$ $0.8+3 \times 17732 \times 0.8=3.9 \times 10^{6}$ many complex numbers) to be held in memory to decide the best suitable estimation; while NN requires to hold the coefficients of designed network $\left(552 \times 128+128 \times 128+128 \times 128+128 \times 64+64 \times 3=1.2 \times 10^{5}\right.$ many real number). In conclusion, the cost of using $\mathrm{NN}$ is to train a neural network, yet after training $\mathrm{NN}$ is more efficient than LUT approach both in terms of time and memory performance.

\section{CONCLUSiON}

In this work, an alternative approach is proposed for obtaining Debye parameters from the reflection coefficient response of the OECP when terminated with a material by utilizing a deep learning model. A synthetically generated dataset was used to design the deep learning model. The synthetic dataset was produced by modeling the OECP with the traditional admittance model [14]. Theoretically modeling the OECP enabled us to effortlessly create a large-scale dataset utilized for designing the deep learning model. A combination of 29 $\epsilon_{s}, 12 \epsilon_{\infty}$ and $51 \tau$ Debye parameters were used to compose Debye models representing a wide range of materials. Among the generated Debye parameters 17732 samples were selected from the generated dataset and reflection coefficients were calculated from 0.5 to $6 \mathrm{GHz}$ with 267 frequency points using the admittance model. A total number of $276 \times 17732$ input dataset (reflection coefficients) and $3 \times 17732$ output dataset (Debye parameters) was produced. To design the deep neural network, 80, 10, $10 \%$ of the dataset was used for training, validating and testing the model, respectively. The model has (mean) \pm (std.dev) percent relative errors between $1.86 \pm 3.01$, $3.33 \pm 9.52$ and $2.07 \pm 7.42$ for predicting $\epsilon_{s}, \epsilon_{\infty}$ and $\tau$ of test set $(10 \%$ of the generated dataset), respectively. In order to test the designed deep learning model with experimental results, 625 input dataset (reflection coefficients) was prepared using measurements collected from five different standard liquids, four mixtures and a gel-like material. Collected measurements were performed in the frequency range of 0.5 to $6 \mathrm{GHz}$ with 20 $\mathrm{MHz}$ resolution. The complex dielectric constants are obtained from the Debye parameters retrieved using the optimized deep learning model and the calculated complex permittivities were compared with reference data obtained from the literature. Obtained minimum and maximum (mean) \pm (std.dev) complex permittivity percent relative errors are $1.21 \pm 0.06$ and $10.89 \pm 0.08$, respectively.

\section{REFERENCES}

[1] M. A. Stuchly and S. S. Stuchly, "Coaxial line reflection methods for measuring dielectric properties of biological substances at radio and microwave frequencies-a review," IEEE Transactions on Instrumentation and Measurement, vol. 29, no. 3, pp. 176-183, 1980.

[2] D. V. Blackham and R. D. Pollard, "An improved technique for permittivity measurements using a coaxial probe," IEEE Transactions on Instrumentation and Measurement, vol. 46, no. 5, pp. 1093-1099, 1997.

[3] M. Lazebnik, L. McCartney, D. Popovic, C. B. Watkins, M. J. Lindstrom, J. Harter, S. Sewall, A. Magliocco, J. H. Booske, M. Okoniewski, et al., "A large-scale study of the ultrawideband microwave dielectric properties of normal breast tissue obtained from reduction surgeries," Physics in medicine \& biology, vol. 52, no. 10, p. 2637, 2007.

[4] B. Filali, F. Boone, J. Rhazi, and G. Ballivy, "Design and calibration of a large open-ended coaxial probe for the measurement of the dielectric properties of concrete," IEEE Transactions on Microwave Theory and Techniques, vol. 56, no. 10, pp. 2322-2328, 2008.

[5] S. A. Komarov, A. S. Komarov, D. G. Barber, M. J. Lemes, and S. Rysgaard, "Open-ended coaxial probe technique for dielectric spectroscopy of artificially grown sea ice," IEEE Transactions on Geoscience and Remote Sensing, vol. 54, no. 8, pp. 4941-4951, 2016.

[6] T. Yilmaz and F. Ates Alkan, "In vivo dielectric properties of healthy and benign rat mammary tissues from $500 \mathrm{mhz}$ to $18 \mathrm{ghz}$," Sensors, vol. 20, no. 8, p. 2214, 2020.

[7] Keysight Technologies, "Keysight n1501a dielectric probe kit $10 \mathrm{mhz}$ to 50 ghz," online; accessed 22 January 2019. [Online]. Available: http://literature.cdn.keysight.com/litweb/pdf/5989-0222EN.pdf

[8] D. Popovic, L. McCartney, C. Beasley, M. Lazebnik, M. Okoniewski, S. C. Hagness, and J. H. Booske, "Precision open-ended coaxial probes for in vivo and ex vivo dielectric spectroscopy of biological tissues at microwave frequencies," IEEE Transactions on Microwave Theory and Techniques, vol. 53, no. 5, pp. 1713-1722, 2005.

[9] J. Grant, R. Clarke, G. Symm, and N. M. Spyrou, "A critical study of the open-ended coaxial line sensor technique for $\mathrm{rf}$ and microwave complex permittivity measurements," Journal of Physics E: Scientific Instruments, vol. 22, no. 9, p. 757, 1989. 
[10] M. A. Stuchly, M. M. Brady, S. S. Stuchly, and G. Gajda, "Equivalent circuit of an open-ended coaxial line in a lossy dielectric," IEEE Transactions on instrumentation and Measurement, no. 2, pp. 116-119, 1982.

[11] T. W. Athey, M. A. Stuchly, and S. S. Stuchly, "Measurement of radio frequency permittivity of biological tissues with an open-ended coaxial line: Part i," IEEE Transactions on Microwave Theory and Techniques, vol. 30, no. 1, pp. 82-86, 1982.

[12] G. Gajda and S. S. Stuchly, "An equivalent circuit of an open-ended coaxial line," IEEE transactions on instrumentation and measurement, vol. 32, no. 4, pp. 506-508, 1983.

[13] F. Monsefi, M. Otterskog, and S. Silvestrov, "Direct and inverse computational methods for electromagnetic scattering in biological diagnostics," arXiv preprint arXiv:1312.4379, 2013.

[14] H. Levine and C. H. Papas, "Theory of the circular diffraction antenna," Journal of applied physics, vol. 22, no. 1, pp. 29-43, 1951.

[15] D. K. Misra, "A quasi-static analysis of open-ended coaxial lines (short paper)," IEEE Transactions on Microwave Theory and Techniques, vol. 35, no. 10, pp. 925-928, 1987.

[16] Y. Xu, R. Bosisio, and T. Bose, "Some calculation methods and universal diagrams for measurement of dielectric constants using open-ended coaxial probes," in IEE Proceedings $H$ (Microwaves, Antennas and Propagation), vol. 138, no. 4. IET, 1991, pp. 356-360.

[17] M. Artioli, M. D. Perez, U. Reggiani, and L. Sandrolini, "Particle swarm optimization method for complex permittivity extraction of dispersive materials," in 2010 Asia-Pacific International Symposium on Electromagnetic Compatibility. IEEE, 2010, pp. 900-903.

[18] N. Marcuvitz, Waveguide handbook. Iet, 1951, no. 21.

[19] M. Li, R. Guo, K. Zhang, Z. Lin, F. Yang, S. Xu, X. Chen, A. Massa, and A. Abubakar, "Machine learning in electromagnetics with applications to biomedical imaging: A review," IEEE Antennas and Propagation Magazine, vol. 63, no. 3, pp. 39-51, 2021.

[20] J. Jin, C. Zhang, F. Feng, W. Na, J. Ma, and Q.-J. Zhang, "Deep neural network technique for high-dimensional microwave modeling and applications to parameter extraction of microwave filters," IEEE Transactions on Microwave Theory and Techniques, vol. 67, no. 10, pp. 4140-4155, 2019.

[21] J. Bonello, A. Demarco, I. Farhat, L. Farrugia, and C. V. Sammut, "Application of artificial neural networks for accurate determination of the complex permittivity of biological tissue," Sensors, vol. 20, no. 16, p. 4640,2020

[22] X. Chen, Z. Wei, M. Li, and P. Rocca, "A review of deep learning approaches for inverse scattering problems (invited review)," Progress In Electromagnetics Research, vol. 167, pp. 67-81, 2020.

[23] "OVERVIEW SPEAG, Schmid \& Partner Engineering AG," swiss. [Online]. Available: https://speag.swiss/products/dak/overview/

[24] B. Jordan, R. Sheppard, and S. Szwarnowski, "The dielectric properties of formamide, ethanediol and methanol," Journal of Physics D: Applied Physics, vol. 11, no. 5, p. 695, 1978.

[25] A. Gregory and R. Clarke, "Tables of the complex permittivity of dielectric reference liquids at frequencies up to $5 \mathrm{ghz}$; npl report mat 23," Innovation, pp. 1-87, 2009.

[26] F. Buckley and A. Maryott, "Tables of dielectric dispersion," Data for pure liquids and dilute solutions, United States Department of Commerce, National Bureau of Standards, Circular, vol. 589, 1958.

[27] T. Mosavirik, M. Soleimani, V. Nayyeri, S. H. Mirjahanmardi, and O. M. Ramahi, "Permittivity characterization of dispersive materials using power measurements," IEEE Transactions on Instrumentation and Measurement, vol. 70, pp. 1-8, 2021.

[28] J. Lou, T. A. Hatton, and P. E. Laibinis, "Effective dielectric properties of solvent mixtures at microwave frequencies," The Journal of Physical Chemistry A, vol. 101, no. 29, pp. 5262-5268, 1997.

[29] J. Guo-Zhu and Q. Jie, "Dielectric constant of dimethyl sulfoxidemonohydric alcohol mixture solution at the microwave frequency," Fluid Phase Equilibria, vol. 365, pp. 5-10, 2014.

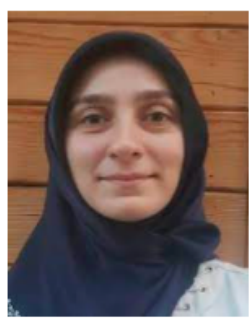

Cemanur Aydinalp received the B.S. degree from the Department of Electronics Engineering, Ankara University, Ankara, Turkey in 2011. The M.S. degree from the department of Electrical and Computer Engineering, San Diego State University, San Diego, USA in 2015 .

Currently she is a Ph.D. candidate in Department of Telecommunication Engineering, Istanbul Technical University, Istanbul, Turkey. Her research interests microwave dielectric spectroscopy, data analysis, optimization of open-ended coaxial probes, application of supervised machine learning algorithms to engineering problems.

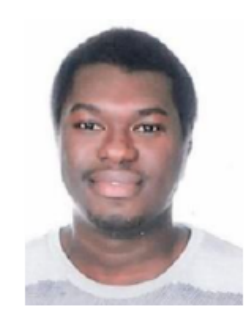

Sulayman Joof Sulayman Joof received the B.S. degree from Department of Electronics and Telecommunication Engineering, Istanbul Technical University, Istanbul, Turkey, in 2015. The M.S. degree from Department of Satellite Communication and Remote Sensing, Istanbul Technical University, Istanbul, Turkey.

Curently, he is working towards his Ph.D. degree in the same Department. His research interests include antenna design, microwave dielectric wireless RF power transfer. spectroscopy, microwave imaging, data analysis, and

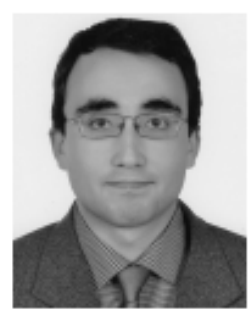

Mehmet Nuri Akinci was born in Ankara, Turkey, in 1992. He received the B.Sc. (Hons.) and Ph.D. degrees in electronics and communication engineering from Istanbul Technical University, Istanbul, Turkey, in 2013 and 2017, respectively.

He was a Visiting Scientist with the Institute for the Electromagnetic Sensing of the Environment, National Research Council of Italy, Naples, Italy, in 2015, and Virginia Commonwealth University, Richmond, VA, USA, in 2016. He is currently an Assistant Professor with the Electronics and Communication Engineering Department, Istanbul Technical University. His current research interests include inverse scattering problems, microwave measurement systems, and optics.

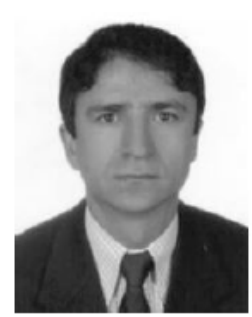

Ibrahim Akduman was born in Konya, Turkey, in 1963. He received the B.Sc., M.S., and Ph.D. degrees in electronics and communication engineering from Istanbul Technical University, Istanbul, Turkey, in 1984, 1987, and 1990, respectively.

He was a Visiting Scientist with the New York University Tandon School of Engineering, Brooklyn, NY, USA, in 1991; King's College London, London, U.K., in 1995; the New Jersey Institute of Technology, Newark, NJ, USA, in 2000; and the University of Göttingen, Göttingen, Germany, in 2001. He was the Dean of the Electrical and Electronics Engineering Faculty, Istanbul Technical University, from 1999 to 2001, and a Vice President from 2002 to 2004. He is currently with Istanbul Technical University, as a Full Professor, where he is also the Head of the Electromagnetic Research Group. His current research interests include microwave tomography and electromagnetics in medicine. He is also a shareholder of a company, where he is involved in research and developing products for medical application of electromagnetic fields. Prof. Akduman received the Turkish Scientific and Technological Research Council Young Scientist Award in 2000. 


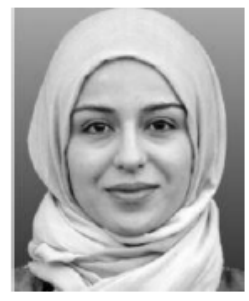

Tuba Yilmaz (S'12-M'14)received the B.S. degree from Istanbul Technical University, Istanbul, Turkey, in 2007, the M.S. degree from Mississippi State University, Mississippi State, MS, USA, in 2009, and the $\mathrm{Ph} . \mathrm{D}$. degree in electronic engineering and computer science from Queen Mary, University of London, London, U.K., in 2013.

She is currently an Assistant Professor and a Marie Sklodowska Curie Research Fellow in Department of Electronics and Communication Engineering at Istanbul Technical University (ITU). Prior to her appointment at ITU, she spent a year at Mitos Medical Technologies as an Associate Research Fellow. From 2013-2014, Dr. Yilmaz was with Utah State University (USU) as a Postdoctoral Research Fellow. Dr. Yilmaz is a member of Eta Kappa Nu Electrical and Computer Engineering Honor Society. She has received URSI Young Scientist Award in 2017. Her research interests include wearable and implantable antennas, RF sensing, dielectric spectroscopy, evolutionary optimization techniques, wireless power transfer, and microwave imaging. 